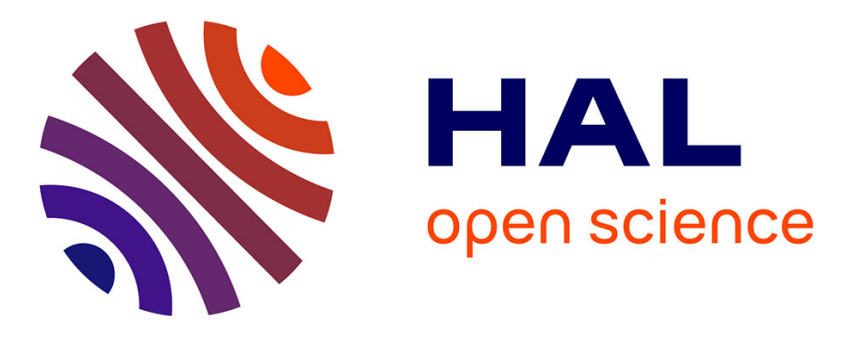

\title{
Recent developments in high speed imaging and applications in speckle light
}

Pierre Slangen, Zacaria Essaïdi, Clement Chanut, Pierre Lauret, Frederic Heymes, Laurent Aprin

\section{- To cite this version:}

Pierre Slangen, Zacaria Essaïdi, Clement Chanut, Pierre Lauret, Frederic Heymes, et al.. Recent developments in high speed imaging and applications in speckle light. SPECKLE 2018: VII International Conference on Speckle Metrology, Sep 2018, Janów Podlaski, Poland. pp.36, 10.1117/12.2318699 . hal-02011901

\section{HAL Id: hal-02011901 \\ https://hal.science/hal-02011901}

Submitted on 8 Jun 2021

HAL is a multi-disciplinary open access archive for the deposit and dissemination of scientific research documents, whether they are published or not. The documents may come from teaching and research institutions in France or abroad, or from public or private research centers.
L'archive ouverte pluridisciplinaire HAL, est destinée au dépôt et à la diffusion de documents scientifiques de niveau recherche, publiés ou non, émanant des établissements d'enseignement et de recherche français ou étrangers, des laboratoires publics ou privés. 


\title{
RECENT DEVELOPMENTS IN HIGH SPEED IMAGING AND APPLICATIONS IN SPECKLE LIGHT
}

\author{
Pierre R. L. Slangen, Z. Essaidi, C. Chanut, P. Lauret, F. Heymes, L. Aprin \\ IMT mines Ales LGEI, Université de Montpellier, France
}

Corresponding Author: pierre.slangen@mines-ales.fr

\begin{abstract}
High speed imagers record images at much higher speed than perceived by the human eye, but also enable to analyze it in different time bases. Recording is the keystone of sensor. It can either be embedded or remoted. The advantage of the onboard system mainly relies on the transfer speed to the in situ memory (including at the photon to charge conversion site). Its major drawback can be the onboard memory size limit. Remote storage requires the transfer of information very quickly to networks of high speed discs. If the main advantage lies in virtually infinite memory size, major drawback is the transfer speed between the camera and the external memory device. Choosing an appropriate high speed camera must be done by selecting, the maximum frame per second rate, minimum exposure time versus sensitivity and maximum recording time versus resolution and speed. Some imagers can now lead to $7 \mathrm{kfps}$ in relatively large resolution to $20 \mathrm{kfps}$ for reduced 1 Mpixel images. Optics and light sources are important as continuous light require freezing the object movement by the camera exposure time, while pulsed source will remove the motion blur. For imaging, pulsed laser source in uncoherent radiation can even be used. Aperture of the optical system will determine speckle size or depth of field. Most of the imagers can be employed lensless for digital holography purposes. Small sensitive pixel will then be very attractive for this. This paper presents the recent developments and application in speckle light.
\end{abstract}

KEYWORDS: high speed imaging, shadowgraphy, schlieren, PIV, digital holography, speckle interferometry

\section{INTRODUCTION}

\subsection{State of the art}

High speed imaging (HSI) allows recording images at very high speed up to 200Mfps (mega frame per second), but also to render the phenomenon and to analyze it in different time bases ${ }^{1,2}$. Frame rates close to the Mfps have been reached by photographic film rolls, thanks to clever mechanical assembly of the film and optical prisms. But hundreds meters of film were used to record just some images of interest. Moreover the latter had to be chemically developed and finally observed. The first electronic cameras worked with tube amplifier, and scanning slit cameras (streak analog camera). At the beginning of the 21th century, photographic films have been completely replaced by electronic sensors, from streak to full field digital cameras. Nowadays digital sensors commonly replace photographic films, also for art photography. The latest developments in rapid acquisition yield to acquire series of 100 images at about the megapixel resolution for several million images per second (ips). The frame rate of the rapid camera is governed by the throughput of the sensor. Throughput limits are now linked to the electronic architecture of the sensor. The maximal throughput, which is the 
maximal amount of data transferred by second, can be expressed in Bit/s or in pixel/s. There are two different architectures in high speed sensor. In situ image storage sensors (ISIS) fulfills mega high speed by recording the live image nearby the active pixel (buried pixel memory) while distant memory uses multiplexing of the sensor reading. These architectures are now fully compatible with CCD or CMOS sensors. Significant progresses are also underway thanks to the generation of multiplexed connectors (e.g. CoaXPress), coupled with flash (Solid State Device) storage disk. This leads to long recording time e.g. several minutes at $1000 \mathrm{fps}$.

Lots of optical techniques ${ }^{3,4,5,6}$ coping with analogic and electronic recording are now perfectly matching digital recording. Coupling of these can yield to the determination of physico-chemical properties of fluid under investigation ${ }^{7}$ or the deformation of solids e.g. composite before brutal rupture $^{8}$. The only regret between photographic film and imaging sensor is the lack of resolution of the latter as holographic film could allow up to $3000 \mathrm{p} / \mathrm{mm}$ while brand new high speed sensors reach only $11 \mu \mathrm{m}$ pixel size i.e. $45 \mathrm{lp} / \mathrm{mm}$. This is in particular crucial for holography with wide angle scene or large objects.

\subsection{Important parameters in high speed imaging}

The main parameters while choosing a high speed sensor are the maximal frame rate at full resolution and the longest recording time. The maximum frame rate is linked to the throughput and the resolution while the recording time relates to the memory size.

The high speed sensor spatial resolution drives the final image quality in terms of resolution and/or localization of defects or deformation. It is directly linked to the pixel size and the pixel pitch...and of course to the total number of pixels (lines and rows). Complex architectures lead to low fill factor and in particular for mega high speed sensors where the live image is "electron-speed" stored in the vicinity memory (memory-stacked so as for Exmor CMOS Sony sensor). Minimal exposure time of about $5 \mathrm{~ns}$ is reached for these sensors and sometimes thanks to image intensifiers. For large rolling memory buffer camera, the minimal exposure time is about 300ns which is compatible with the $1 \mathrm{Mfps}$ maximum frame rate achieved for lower resolution at constant throughput.

If the event under investigation is very fast, the movement can be frozen by using pulsed light sources, generally super luminescent LED or in particular multi-pulsed laser. Coherent lasers have to be used for interferometric measurements while uncoherent lasers are preferred for imaging techniques e.g. schlieren, shadowgraphy or correlation techniques (Digital Image Correlation DIC, Particle Image Velocimetry PIV).

Some practical tips can also be pointed out such as download speed of large memory buffer (e.g. 288GB) in special SSD disk, battery-powered camera, size and weight ... and up to single use high speed camera.

\section{HIGH SPEED IMAGING SENSORS}

\subsection{Definitions}

After video speed from 24-100fps, and to extend the definition from Reu and Miller ${ }^{9}$, it is generally accepted to consider high speed imaging from $100 \mathrm{fps}$ to $1000 \mathrm{fps}$, very high speed imaging for $1000 \mathrm{fps}-50 \mathrm{kfps}$ and ultra high or mega high speed for $50 \mathrm{kfps}-200 \mathrm{Mfps}$. The main imaging sensors

used to reach such rates are the CCDs and CMOS sensors ${ }^{10,11}$. Recording frequencies reaching $26000 \mathrm{fps}$ are now performed for full format CMOS 1megapixel images. In some cases, if the full format resolution is decimated by lines and columns, acquisition frequencies of a million thumbnails images per second can be reached.

Typical examples are shown in table 1 for standard format and scientific formats. 
Table1: Typical high speed sensor formats and pixel sizes

\begin{tabular}{|c|c|c|c|c|}
\hline Sensor size & Standard size & \multicolumn{2}{|l|}{ Sensor diagonal } & Pixel size $(H \times V)$ \\
\hline 1 inch & $12.8 \mathrm{~mm} \times 9.6 \mathrm{~mm}$ & \multicolumn{2}{|c|}{$16 \mathrm{~mm}$} & $20 \mu \mathrm{m} \times 20 \mu \mathrm{m}$ \\
\hline $2 / 3$ inch & $8.8 \mathrm{~mm} \times 6.6 \mathrm{~mm}$ & \multicolumn{2}{|c|}{$11 \mathrm{~mm}$} & $13.8 \mu \mathrm{m} \times 13.8 \mu \mathrm{m}$ \\
\hline $1 / 2$ inch & $6.4 \mathrm{~mm} \times 4.8 \mathrm{~mm}$ & \multicolumn{2}{|c|}{$8 \mathrm{~mm}$} & $10 \mu \mathrm{m} \times 10 \mu \mathrm{m}$ \\
\hline $1 / 3$ inch & $4.8 \mathrm{~mm} \times 3.6 \mathrm{~mm}$ & \multicolumn{2}{|c|}{$6 \mathrm{~mm}$} & $7.5 \mu \mathrm{m} \times 7.5 \mu \mathrm{m}$ \\
\hline $1 / 4$ inch & $3.2 \mathrm{~mm} \times 2.4 \mathrm{~mm}$ & \multicolumn{2}{|c|}{$4 \mathrm{~mm}$} & $5 \mu \mathrm{m} \times 5 \mu \mathrm{m}$ \\
\hline Camera reference & Sensor size & Pixel area & fps full frame & Pixel size $(H X V)$ \\
\hline \multirow[t]{2}{*}{ Phantom V2512 } & $35.8 \mathrm{~mm} \times 22.4 \mathrm{~mm}$ & $1280 \times 800$ & 26000 & $28 \mu \mathrm{m} \times 28 \mu \mathrm{m}$ \\
\hline & & $128 \times 32$ & 1000000 & \\
\hline Photron SA-Z & $20.8 \mathrm{~mm} \times 20.5 \mathrm{~mm}$ & $1024 \times 1024$ & 20000 & $20 \mu \mathrm{m} \times 20 \mu \mathrm{m}$ \\
\hline \multirow[t]{2}{*}{ IX i-SPEED 726R } & $27.97 \mathrm{~mm} \times 20.7 \mathrm{~mm}$ & $2048 \times 1536$ & 8512 & $13.5 \mu \mathrm{m} \times 13.5 \mu \mathrm{m}$ \\
\hline & & $336 \times 42$ & 1000000 & \\
\hline PhotonFocus MV1 & $12.8 \mathrm{~mm} \times 9.60 \mathrm{~mm}$ & $1312 \times 1082$ & 108 & $8 \mu \mathrm{m} \times 8 \mu \mathrm{m}$ \\
\hline Phantom Flex 4K & $27.6 \mathrm{~mm} \times 15.5 \mathrm{~mm}$ & $4096 \times 2304$ & 1000 & $6.75 \mu \mathrm{m} \times 6.75 \mu \mathrm{m}$ \\
\hline Shimadzu HPV X2 & $14.4 \mathrm{~mm} \times 8.00 \mathrm{~mm}$ & $200 \times 250-256 f r$ & 10000000 & $32 \mu \mathrm{m} \times 32 \mu \mathrm{m}$ \\
\hline Kirana & $27.7 \mathrm{~mm} \times 23 \mathrm{~mm}$ & $924 \times 768-180 \mathrm{fr}$ & 5000000 & $30 \mu \mathrm{m} \times 30 \mu \mathrm{m}$ \\
\hline
\end{tabular}

Progress is particularly obtained thanks to the advent of new sensors that reach high light sensitivity. This high sensitivity is the sine qua non for achieving super-short shutter time ( 300ns for CMOS) which therefore allows very high acquisition rates (1Mfr/s). Sensitivity of the sensors reaches up to ISO 100000T (Tungsten) regarding ISO (12232-2:2006, D, sat) or EMVA (http://www.emva.org/standards-technology/emva-1288/). Some microlens array can also be placed to enhance the light collection on the sensitive area. The intrinsic sensitivity of the sensors can also be coupled with image intensifiers, despite resolution loss, which amplify the light signal to yield shutter time to the nanosecond. It is also possible to compare the different camera sensors by record length versus frame rate chart ${ }^{12}$.

Figure 1 presents three different cameras from different categories, available for laboratories or industrial sites.
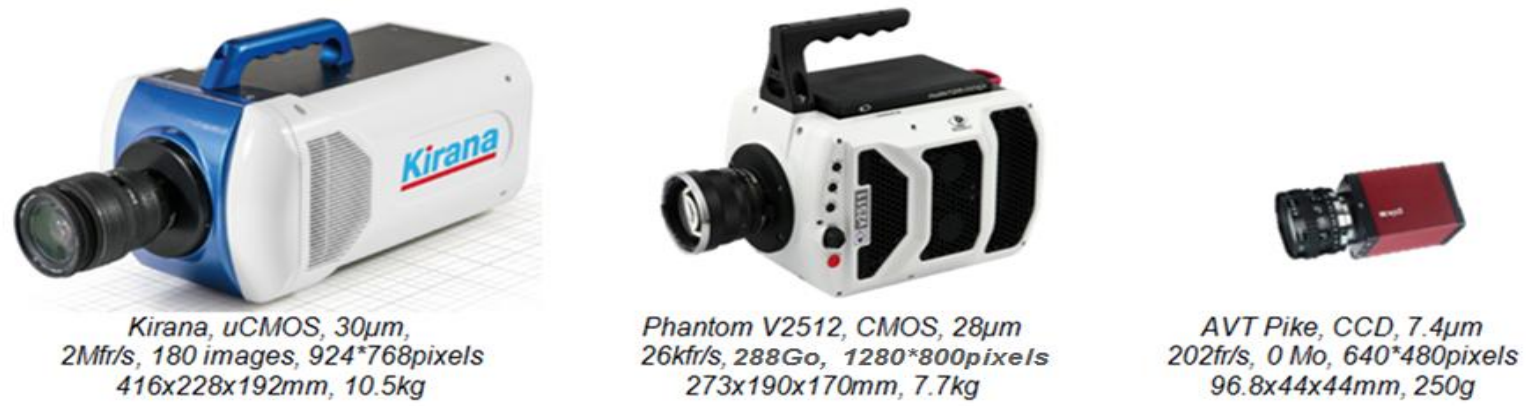

Figure 1: Comparison of performances, dimensions and weight for Kirana, Phantom V2512 and AVT Pike

Nowadays two families of sensors are developed (In Situ Image Storage CCD sensor, CMOS), including some crossing between them such as uCMOS ("ultra-high-speed CMOS") enabling millions fps but for short number of recorded frames (up to 240 stored images) from 8 to 12 bits depth $^{13}$. The main differences are the sensitivity of the pixel sensors as $200 \mathrm{Mfps}$ requires at least $5 \mathrm{~ns}$ time exposure... and so consuming a lot of light (superlum LED, flash, pulsed laser, plasma or HMI lamps) or self-illuminated objects. Ultra-high speed is reached by transferring the full-frame active image area to neighborhood storage image at "electron speed" in ISIS CCD ${ }^{14}$ while CMOS sensors can transfer $\mathrm{ROI}$ active image up to $25 \mathrm{~GB} / \mathrm{s}$ in on-board memory up to $288 \mathrm{~GB}$. The lacks for UHS sensors are 3 
commonly the low fill factor (e.g. 10\% for ISIS) and potential blooming of surrounding stored images. For CMOS sensors, fixed pattern noise and local blooming can occur. Fixed pattern noise can be reduced by applying "black reference or shading". Low noise between images is achieved by correlated double sampling. Sensor is then covered manually or closed by mechanical shutter to reset all amplifiers to zero. Local blooming from highly variable intensity areas can be avoided for some events by using "extended dynamic range EDR" or so-called "dual-slope shutter". The exposure time of high level pixels can then be decreased locally from one image to the next one during recording.

But the best advantage of digital sensor is the recording mode in rolling buffer memory (RMB) or FIFO mode (Figure 3). RMB enables triggering the acquisition from passive (rewriting the buffer in FIFO, pre-trigger) to active or live images (keeping an amount of passive images before trigger and active acquisition after trigger in the post-trigger buffer). Different triggers can also define dedicated memory partitions with different frame rates.

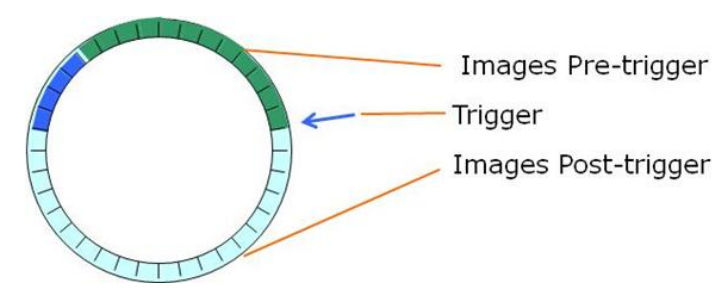

Figure 2: Rolling buffer memory principle

\subsection{Exposure time and compatibility}

As above-mentioned high speed imaging is enabled by short exposure time. So the observed scene must render light enough. In case of luminous objects (sparks, hypervelocity impact, explosion) the luminous phenomenon can even mask the desired measurement (flying debris, crack propagation). The whole scene is then lighten up by powerful system e.g. 4kW HMI projectors or pulsed incoherent laser (e.g. Cavitar@ Cavilux). Superlum LED arrays can also be used to reduce the radiated heat from the illumination device onto the object. The user must then choose through a lot of lighting to cope with the application, keeping in mind that $\mathrm{HSI}$ can even catch the very high speed flickering of some light sources (e.g. halogens light bulbs, luminescent tubes).

For interferometric application, pulsed coherent lasers or CW coherent lasers have to be used. Large depth of field is not relevant in shadowgraphy nor in PIV. Large aperture of fast optics is often used to reach short exposure time. For these imaging techniques, speckle can then be neglected as the pixel size of the high speed sensor is much bigger than the speckle size. Nevertheless some secondary interference fringes can appear from coatings or windows, in particular by viewing phenomena through protective windows in case of explosion.

The other problem dealing with high speed imaging is the profusion of images even for short time lapse... and the evidence to store gigabits of relevant sequences. Moreover the presentation of direct $\mathrm{HSI}$ results is very poor without movie projection in slow motion and space consuming in paper presentation.

With sharp focus and good contrast balance between the target and the background, HSI enables particle tracking velocimetry (PTV) or particle image velocimetry (PIV) including digital image correlation (DIC) or image flow analysis also applied on shadowgraphy or schlieren optical techniques 15,16. These techniques yield to displacement maps showing quantitative information in fluids or solid mechanics.

Finally, $\mathrm{HSI}$ is fully compatible with techniques like speckle interferometry, digital holography ${ }^{17}$ and phase sensitive techniques when the phase distortion from the sensor has been calibrated or the 
sensor protection window has been removed. Another field is also vibration measurements for high frequencies ${ }^{18}$.

\subsection{Triggering and synchronization}

High speed cameras can be associated together to enlarge the field of view in stitching mode for spatial resolution enhancement. Synchronized comparison of dual observation by shadowgraphy and schlieren can also be performed. Acquisition frequencies must be locked from one master camera to slave cameras. Sometimes the time synchronization is even better with Inter-Range Instrumentation Group-B protocol (IRIG-B) timing signal. This stitching can be done linearly or spatially to reach high spatial and temporal resolution. Different viewing angles to complete observation around the object are also of first interest ${ }^{6}$. The main application in mechanical engineering using at least two cameras is stereovision for 2D-3C analysis ${ }^{20,21}$ as in 3D PIV for fluid mechanics, up to plenoptic imaging ${ }^{22}$. But in any cases the cameras have to be sharply "event-triggered" to avoid some delay between the same image pair ${ }^{23}$. Some latency can come from image resetting or straddle time. For UHSI, the main problem is then to synchronize the event detection with the camera trigger....within less than $1 \mu \mathrm{s}$ if the recording speed is $200 \mathrm{Mfps}$ and the memory space is exclusively 180 frames. In this paper, only large rolling buffer memory cameras will be used. The onboard memory enables about 3seconds of recording at nominal resolution/speed e.g. 2000,7500 or $26000 f p s$.

\section{APPLICATIONS IN SPECKLE LIGHT}

In this paper we will describe two applications of high speed sensors in laser light carried on in our lab: imaging with refractive index gradient sensitive methods (direct shadowscopy and schlieren) and interferometry for digital holography. These techniques are applied to dust explosion of aluminum particles and interaction of shockwave with falling droplets, respectively.

\subsection{Shadowgraphy, schlieren and dust explosion}

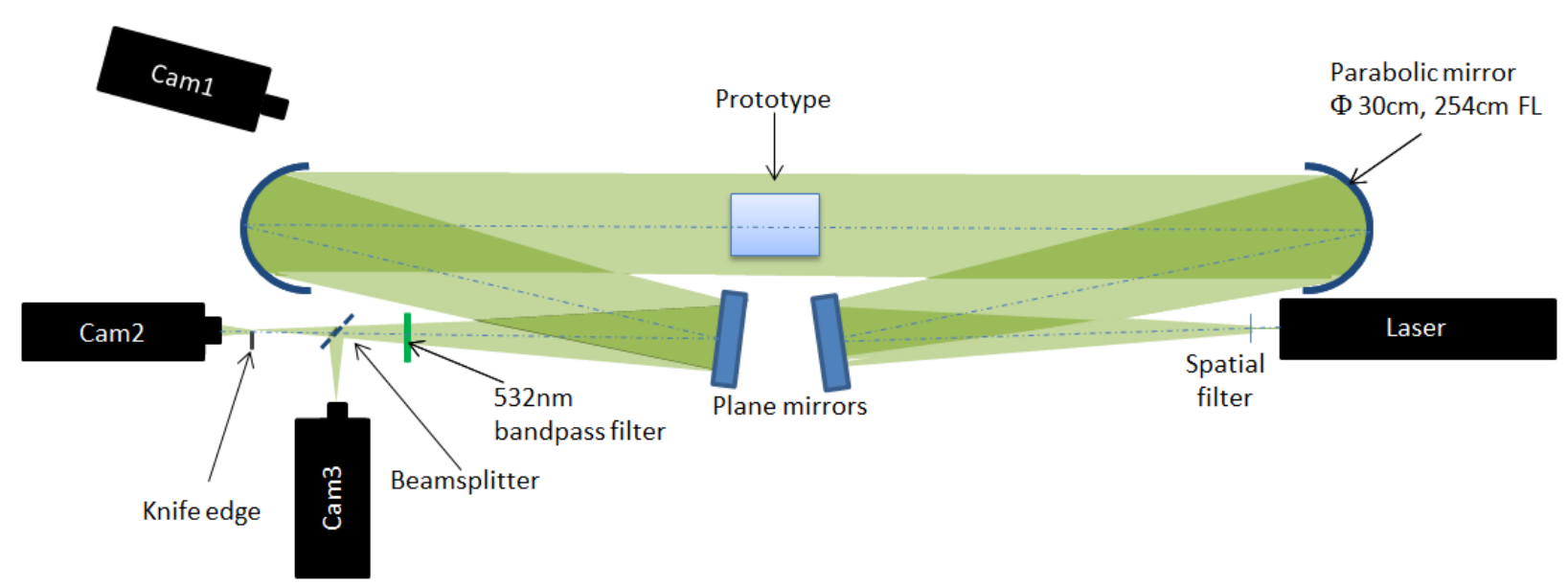

Figure 3: Optical set-up for dust explosion high speed imaging

This section presents our contribution to study of aluminum dust flame propagation ${ }^{24}$ inside a vertical prototype of $700 \mathrm{~mm}$ height and $150 \times 150 \mathrm{~mm}$ squared cross section. This work considers three visualization techniques in high speed. Direct visualization (cam1) is deployed to record the flame light. Special attention is given to collect images without saturation. This is especially important with aluminum flames as they are very luminous. To ensure that the exact delimitation of the flame is well defined, two additional optical techniques have been implemented: high speed schlieren (cam2) and 
shadowgraphy (cam3). These three techniques were used simultaneously to compare the flame propagation. For each test, the tank is filled by blown air carrying aluminum dust of about $6.5 \mu \mathrm{m}$ diameter. The Al powder concentration is $350 \mathrm{~g} \cdot \mathrm{m}^{-3}$. An electric spark then generates $13 \mathrm{~J}$ locally to initiate the dust explosion from the basis to the top of the open prototype equipped with outside exhauster. Recording trigger is done on spark ignition and all the cameras, running at 7500fps, are IRIG locked to ensure the right temporal synchronization of all the images.

The Z-type shadowgraph set-up is common for both schlieren (sensitive to $\mathrm{dn} / \mathrm{dx}$ ) and shadowgraph $\left(d^{2} n / d x^{2}\right)$ cameras. Bright light from the dust explosion is filtered by the band pass filter centered at $532 \pm 10 \mathrm{~nm}$ which is the wavelength of the Verdi $5 \mathrm{~W}$ CW laser. Laser power is adjusted to get laser light as bright as the explosion light passing at 532nm anyway. Before focusing the light is divided by $50 / 50$ beam splitter to cope with common path for cam2 and cam3. The region of interest is $30 \mathrm{~cm}$ wide and imaged with full resolution of the three cameras. The knife edge selects mainly the strong refractive index variations in the vertical direction for schlieren. The same magnification is obtained for each camera by gently calibrating the three devices with the same ruler. The parameters are presented in Table2.

Table 2: Optical and camera parameters

\begin{tabular}{c|cccc|cc} 
Optical Technique & Camera & $\begin{array}{c}\text { Frame rate } \\
\text { fps }\end{array}$ & $\begin{array}{c}\text { Resolution } \\
\text { pixel }\end{array}$ & $\begin{array}{c}\text { Exposure Time (EDR) } \\
\mu \mathrm{s}\end{array}$ & $\begin{array}{c}\text { Zoom } \\
\text { lens }\end{array}$ & $\begin{array}{c}\text { Lens } \\
\text { Aperture }\end{array}$ \\
\hline Cam1: Direct & V2512 & 7500 & $1280^{\star} 800$ & $5 \mu \mathrm{s}(2.5 \mu \mathrm{s})$ & $70-200 \mathrm{~mm}$ & $\mathrm{f} / 32$ \\
Cam2: Schlieren & V711 & 7500 & $1280^{\star} 800$ & $5 \mu \mathrm{s}(2.5 \mu \mathrm{s})$ & $70-300 \mathrm{~mm}$ & $\mathrm{f} / 4$ \\
Cam3: Shadowgraphy & V2512 & 7500 & $1280 \star 800$ & $5 \mu \mathrm{s}(2.5 \mu \mathrm{s})$ & $70-300 \mathrm{~mm}$ & $\mathrm{f} / 4$ \\
\hline
\end{tabular}
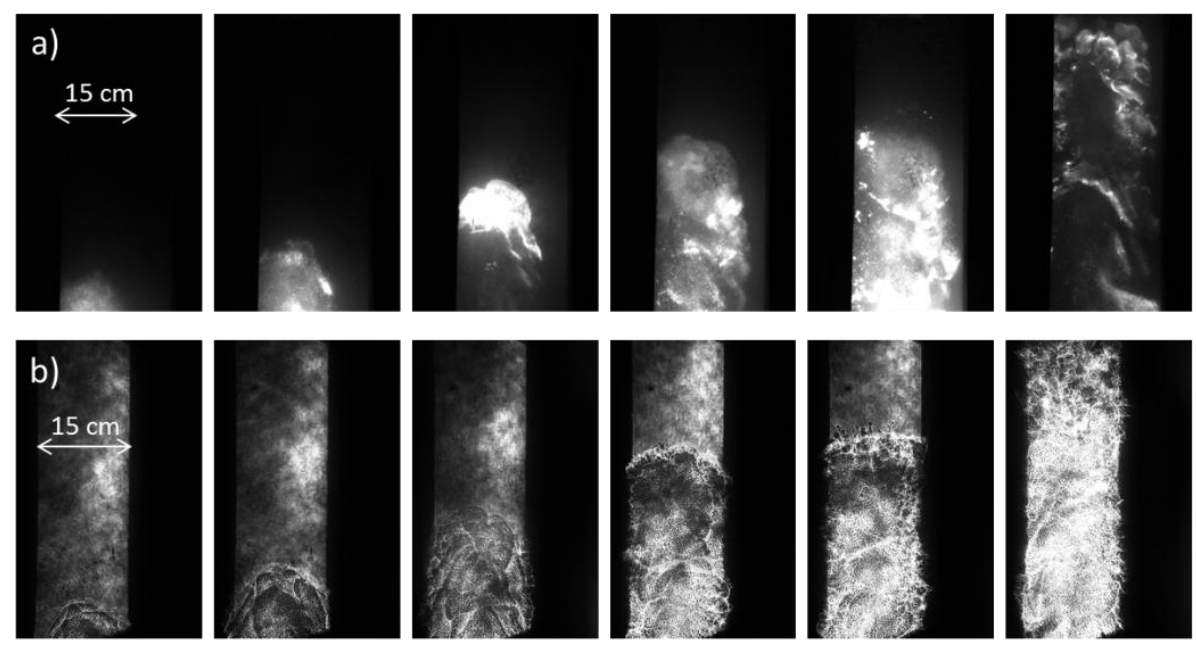

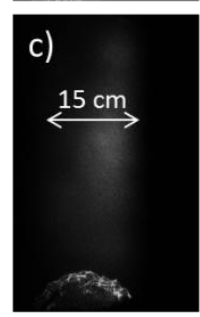

$\mathrm{t}=151,3 \mathrm{~ms}$

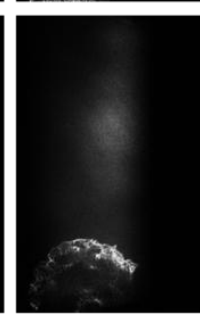

$\mathrm{t}=165,3 \mathrm{~ms}$

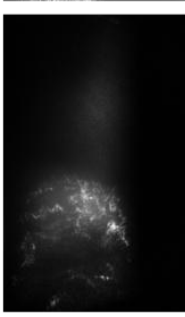

$\mathrm{t}=179,3 \mathrm{~ms}$

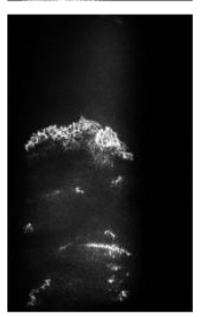

$\mathrm{t}=193,3 \mathrm{~ms}$

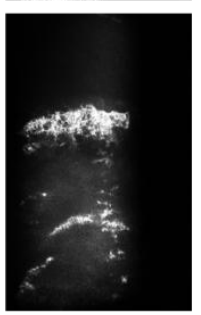

$\mathrm{t}=207,3 \mathrm{~ms}$
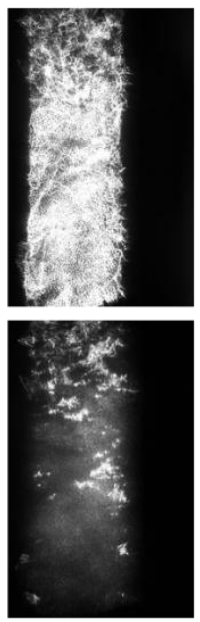

$\mathrm{t}=221,3 \mathrm{~ms}$

Figure 4: Flame propagation velocity on the upper part of the prototype: by direct visualization (a), shadowgraphy (b) and schlieren (c). Time between images: $14 \mathrm{~ms}$

Figure 4 presents the image sequences for the flame propagation. The main problem of direct imaging is then to select the real frontier between the flame and the surrounding particles illuminated by the 
flame. This produces a "milky" transition zone difficult to interpret by simple thresholding. Schlieren and shadowgraphy images show the flame temperature effect on the refractive index derivatives. So there are no more side effects of the particles backscattered light. Figure 5 presents the evolution of the flame front position after edge processing (Canny filter).

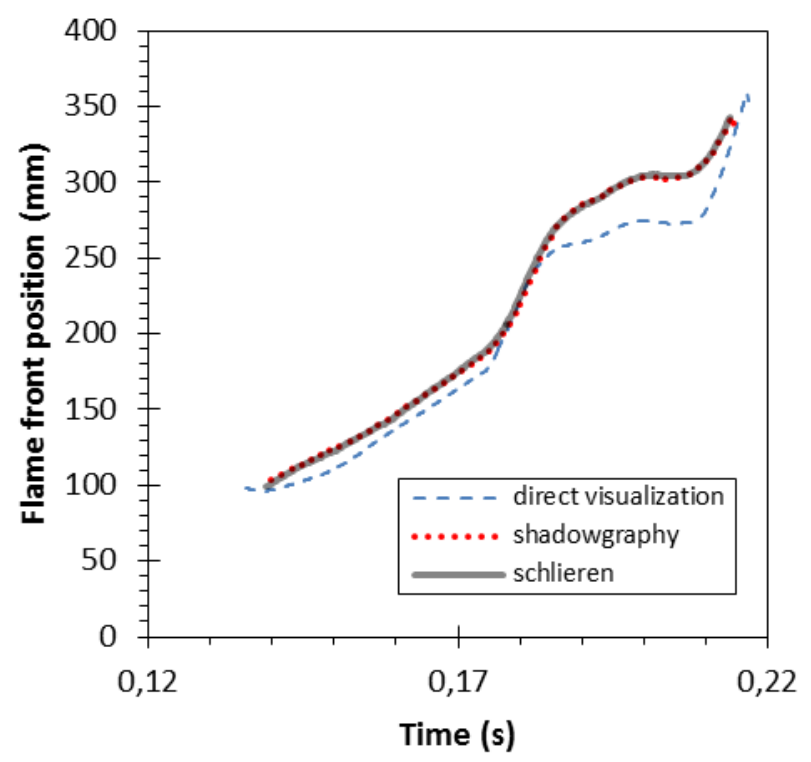

Figure 5: Evolution of the flame front position for the upper zone of visualization

Both schlieren and shadowgraph results are consistent. It is clearly seen the flame is accelerating after $0.18 \mathrm{~s}$. To simplify set-up and alignment, shadowgraphy will be used for further experiences. These data will then allow computing the propagation velocity and finally the estimation of the burning velocity thanks to longer experiment tube ensuring a less pulsatile flame.

\subsection{Digital holography of falling droplets}

Falling droplets have been studied successfully by different authors ${ }^{25},{ }^{26}$. In this study, droplets of liquids with controlled physico-chemical properties are falling in front of the open end of short shock tube. This tube generates a shockwave interacting with the droplets. As measured by technique of section 3.1 , shockwave speed is about $380 \mathrm{~m} / \mathrm{s}$ and can be considered planar at the droplet scale. The fragmentation is studied by time-resolved digital in-line holographic interferometry TRDILHI (Figure 6).

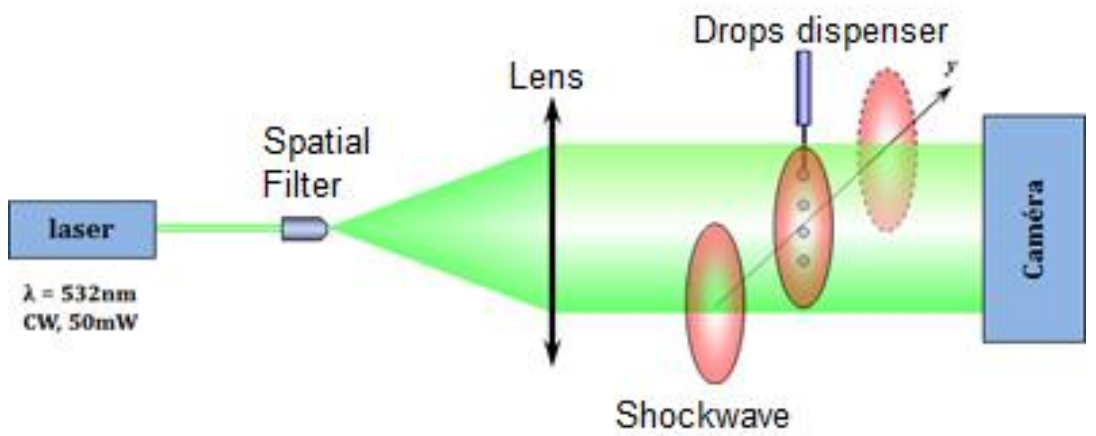

Figure 6: Set-up for in-line digital holography on falling droplets with shockwave.

The continuous coherent source emitting at a wavelength of $532 \mathrm{~nm}$ with a maximum power of $50 \mathrm{~mW}$ (CNI laser) is collimated after spatial filtering and impinges the high speed CMOS camera (Phantom 
VEO 710 camera). The VEO 710 presents $20 \mu \mathrm{m}$ squared pixels. Full resolution frame rate is $7400 \mathrm{fps}$ and must be adjusted to $24000 \mathrm{fps}$ to catch the shockwave across the $10.2 \mathrm{~mm}$ wide region of interest within $512 \times 512$ pixel $^{2}$. Exposure time is $1 \mu$ s to freeze the secondary droplets. The distance between the lens less camera and the droplets must be large enough to avoid damage by the shockwave and short enough to ensure a good sampling of the hologram ${ }^{27,28}$. Considering the pixel size and pitch, the minimal distance is about $33.6 \mathrm{~cm}$ between the sensors and the droplets.
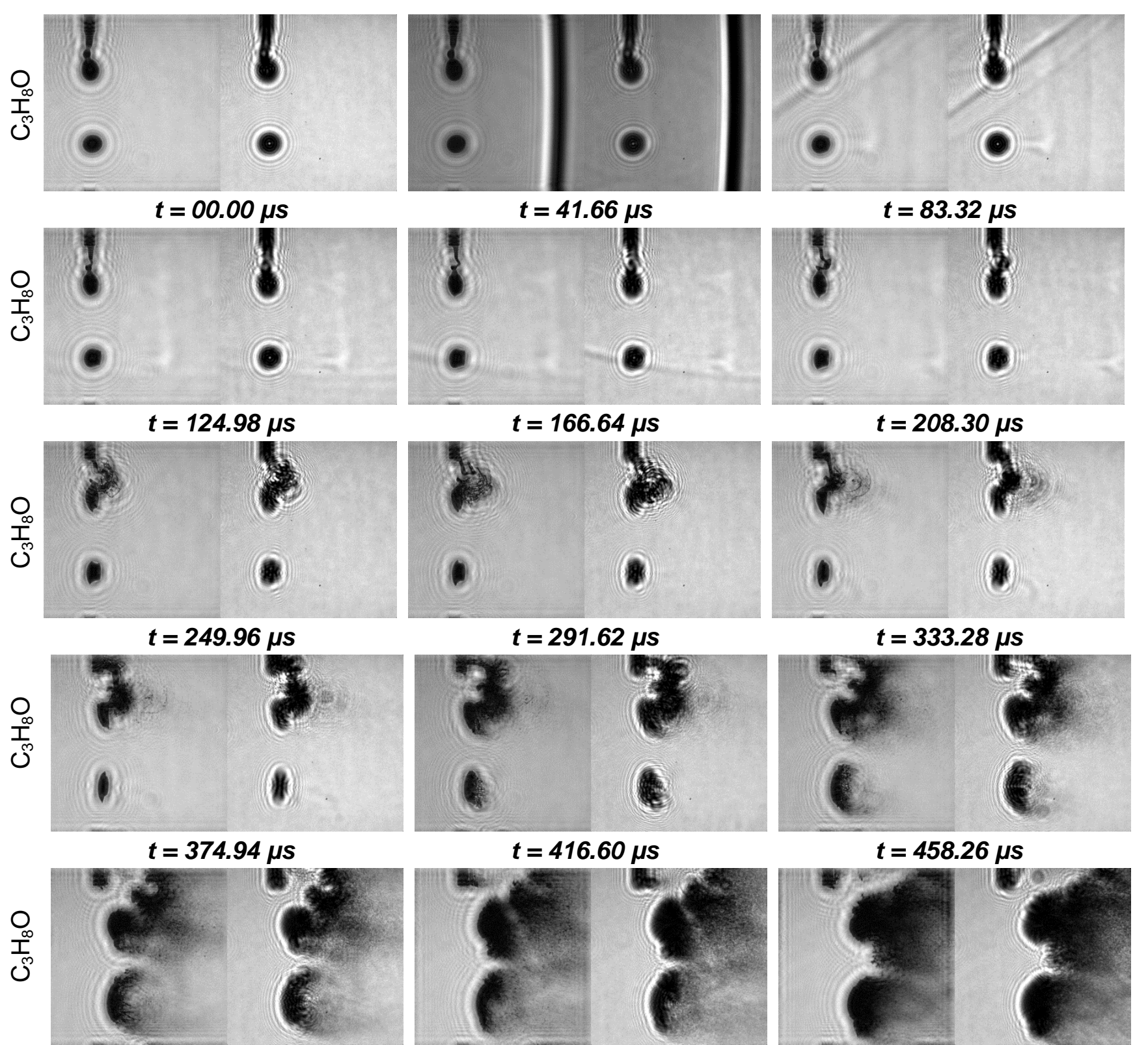

$$
t=499.92 \mu \mathrm{s}
$$

$$
t=541.58 \mu \mathrm{s}
$$
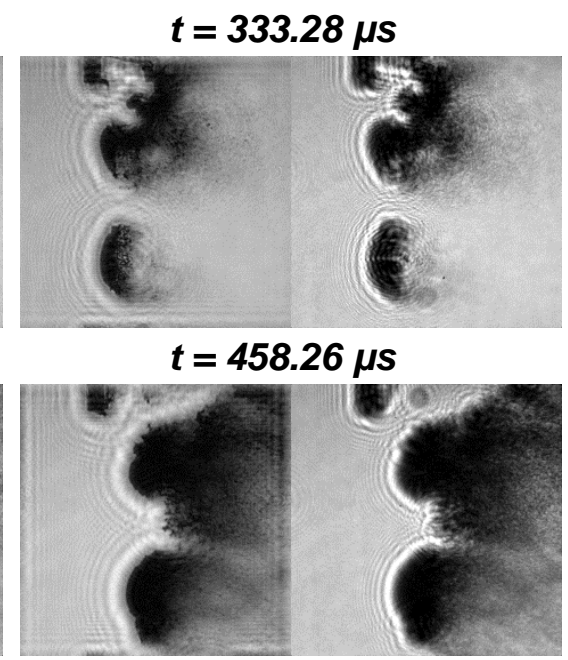

$$
t=583.24 \mu \mathrm{s}
$$

Figure 7: TRILDHI Time code images for ethanol: unitary magnification 20 $\mu \mathrm{m} /$ pixel, 512x512pixels, 24890fps

The camera is free running and waiting for trigger after the generation of the shockwave from the tube outlet. The shockwave characteristics have been previously studied by shadowscopy (see section 3.1). Recorded holograms are then back propagated via MATLAB program based on the angular spectrum propagation. TRDILHI enables to numerically refocus many particles along the optical depth. Moreover it is also possible to reconstruct 3D liquid ligaments with wide depth of field or to detect particles about $10 \mu \mathrm{m}$ over the $1.02 \mathrm{~cm}$ wide ROI. Different liquids (ethanol, propanol, water...) have 
been tested to change the physico-chemical properties and so the fragmentation process. The results of reconstructed and raw images for pure isopropanol are shown figure 7 . The shockwave, running at about $380 \mathrm{~m} / \mathrm{s}$ from left to right (bright left side), is clearly shown at $41.66 \mu \mathrm{s}$. Some artifacts due to inline configuration are still present. Speed and size of the secondary droplets are also in very good agreement with previous ones ${ }^{7}$.

\section{CONCLUSION}

High speed imaging is now present in lots of laboratory or even onboard drones. The amount of recorded images and the throughput to transfer it are now the bottleneck to achieve further developments. Multiplexing high speed imagers can lead to plenoptic high speed imaging, with an increased cost of the set-up. More recently, $1 \mathrm{Tfps}$ have been reached by sequentially timed all-optical mapping photography (STAMP) ${ }^{29}$.

Moreover ultrafast imaging perfectly match with optical systems e.g. shadowscopy, schlieren or holographic and speckle interferometry ....and so generating incredible slow motion replays to see and then to analyze even invisible phase modulations. All these means give scientists and engineers the opportunity to sharply study materials and systems of the future, linked to these new developments.

We have shown the applications of HSI in speckle light, as for imaging or for interferometric purposes. The derived results are then obtained from computer analysis of the different images. For large number of images, parallel computing and GPU processing will shorten the processing time and probably allow real time computing in the next few years. On board HSI systems with post processing unit coupled to dedicated optical set-up will then equip brand new autonomous vehicles to reinforce safety and may be security of the users.

But the most amazing effect of $\mathrm{HSI}$ and UHSI is to allow the user to create amazing slow motion helping him/her to retrieve or model temporally hidden information.

\section{REFERENCES}

[1] RAY, F. S., [High Speed Photography and Photonics], SPIE Press, Bellingham, WA (2002).

[2] Versluis, M., "High-speed imaging in fluids," Exp. Fluids 54(2) (2013).

[3] Jacquot, P., Slangen, P. and Borza, D., [Elementary Speckle Interferometry] (2012).

[4] Settles, G. S. and Hargather, M. J., "A review of recent developments in schlieren and shadowgraph techniques," Meas. Sci. Technol. 28(4), 042001 (2017).

[5] Tropea, C., Yarin, A. L. and Foss, J. F., [Springer handbook of experimental fluid mechanics], Springer Science+Business Media (2007).

[6] Rastogi, P. K. and Hack, E., [Optical methods for solid mechanics : a full-field approach], Wiley$\mathrm{VCH}$ (2012).

[7] Slangen, P. R., Lauret, P., Heymes, F., Aprin, L. and Lecysyn, N., "High-speed imaging optical techniques for shockwave and droplets atomization analysis," Opt. Eng. 55(12) (2016).

[8] Puech, L., Ramakrishnan, K. R., Le Moigne, N., Corn, S., Slangen, P. R., Duc, A. Le, Boudhani, $\mathrm{H}$. and Bergeret, A., "Investigating the impact behaviour of short hemp fibres reinforced polypropylene biocomposites through high speed imaging and finite element modelling," Compos. Part A Appl. Sci. Manuf. 109, 428-439 (2018).

[9] Reu, P. L. and Miller, T. J., "The application of high-speed digital image correlation," J. Strain Anal. Vol. 43(Special Issue), 673-688 (2008).

[10] HOLST, G. C. and LOMHEIM, T. S., [CMOS/CCD Sensors and Camera Systems] (2011).

[11] Durini, D., [High performance silicon imaging: fundamentals and applications CMOS and CCD sensors], Elsevier (2014).

[12] Reu, P. L. and Nissen, M., "The evolution of high and ultra-high speed imaging from qualitative to quantitative," Proc. BSSM (2014).

[13] Crooks, J., Marsh, B., Turchetta, R., Taylor, K., Chan, W., Lahav, A. and Fenigstein, A., "Kirana: a solid-state megapixel uCMOS image sensor for ultrahigh speed imaging," 19 February 2013, 
865903, International Society for Optics and Photonics.

[14] Etoh, T. G., Dao, V. T. S., Nguyen, H. D., Fife, K., Kureta, M., Segawa, M., Arai, M. and Shinohara, T., "Progress of Ultra-high-speed Image Sensors with In-situ CCD Storage," Int. Image Sens. Work., 1-4 (2011).

[15] Andrieux, S., "Inverse Problems and Experiments : a Fruitful Symbiosis," J. Aerosp. Lab(12), 112 (2016).

[16] Mauger, C., Méès, L., Michard, M. and Lance, M., "Velocity measurements based on shadowgraph-like image correlations in a cavitating micro-channel flow," Int. J. Multiph. Flow 58, 301-312 (2014).

[17] Desse, J. M. and Olchewsky, F., "Digital Holographic Interferometry for Analysing High- Density Gradients in Fluid Mechanics," [Holographic Materials and Optical Systems pumped], 291-318 (2017).

[18] Picart, P., Poittevin, J., Gautier, F. and Pezerat, C., "High-speed holographic metrology: Principle, limitations and application to vibro-acoustics of structures," Opt. Eng. 55(12), 121717 (2016).

[19] Slangen, P. R., Lauret, P., Heymes, F., Aprin, L. and Lecysyn, N., "High-speed imaging optical techniques for shockwave and droplets atomization analysis," Opt. Eng. 55(12) (2016).

[20] Orteu, J.-J., "3-D computer vision in experimental mechanics," Opt. Lasers Eng. 47(3), 282-291 (2009).

[21] Siebert, T., "High-speed digital image correlation: error estimations and applications," Opt. Eng. 46(5), 051004 (2007).

[22] Wetzstein, G., Ihrke, I., Lanman, D. and Heidrich, W., "Computational plenoptic imaging," Comput. Graph. Forum 30(8), 2397-2426 (2011).

[23] Reu, P. L. and Miller, T. J., "Synchronization Errors in High-Speed Digital Image Correlation," Proc. SEM, SEM, Ed., Albuquerque (2009).

[24] Gao, W., Mogi, T., Yu, J., Yan, X., Sun, J. and Dobashi, R., "Flame propagation mechanisms in dust explosions," J. Loss Prev. Process Ind. 36, 186-194 (2014).

[25] Seifi, M., Fournier, C., Grosjean, N., Méès, L., Marié, J.-L. and Denis, L., "Accurate 3D tracking and size measurement of evaporating droplets using in-line digital holography and inverse problems' reconstruction approach," Opt. Express 21(23), 27964 (2013).

[26] Guildenbecher, D. R., Engvall, L., Gao, J., Grasser, T. W., Reu, P. L. and Chen, J., "Digital inline holography to quantify secondary droplets from the impact of a single drop on a thin film," Exp. Fluids 55(3) (2014).

[27] Picart, P., Karray, M. and Slangen, P., "Some considerations about the role of the diaphragm in digital image-plane holography," Digit. Hologr. Three-Dimensional Imaging, DH 2012 (2012).

[28] Sentis, M. P. L., Bruel, L., Charton, S., Onofri, F. R. A. and Lamadie, F., "Digital in-line holography for the characterization of flowing particles in astigmatic optical systems," Opt. Lasers Eng. 88(January), 184-196 (2017).

[29] Mikami, H., Gao, L. and Goda, K., "Ultrafast optical imaging technology: Principles and applications of emerging methods," Nanophotonics 5(4), 441-453 (2016). 